\title{
Application of field inspections and odour observation diaries in the assessment of air quality and odour in urban areas
}

\author{
Izabela Sówka ${ }^{1,{ }^{*}}$ Urszula Miller ${ }^{1}$, Yaroslav Bezyk ${ }^{1}$, Alicja Nych $^{1}$, Agnieszka Grzelka ${ }^{1}$, and \\ Łukasz Dąbrowski ${ }^{1}$ \\ ${ }^{1}$ Wroclaw University of Science and Technology, Faculty of Environmental Engineering, \\ Pl. Grunwaldzki 9, 50-377 Wroclaw, Poland
}

\begin{abstract}
The choice of an odour nuisance assessment method depends on many factors, such as: the type and quantity of objects, the diversity and type of odour sources, the location of the object, the social situation and the number of complaints about odor nuisance, the type and physicochemical parameters of the emitted gases and the conditions of dispersion of pollutants in the air. In most cases, in order to fully assess the situation and take integrated actions to reduce odour nuisance, both an instrumental/sensory analysis and a sociological research method should be used simultaneously. Such an approach enables researchers to get a real picture of the problem and develop the optimal and necessary minimization of odour impact solutions. The work presents the results of field and sociological research aimed at identifying odour sources and odour nuisance in a selected urban area located in the southern part of Poland. The studied area is complex in terms of the type and amount of odour emission sources, hence the use of several methods in the assessment of air quality was justified. The conducted research allowed the identification of emission sources and the extent of their impact on the area of the examined city and community.
\end{abstract}

\section{Introduction}

The assessment of the olfactory air quality is a very complex problem. This fact is caused by many factors which affect the appearance of the smell, its sensation and ultimately the cause of the nuisance. The most important factors include the type of the source, its location and the number of objects emitting odours, as well as a group of social factors, not directly related to odour [1-3]. The latter are the so called non-olfactory factors, i.e. socio-economic (e.g. place of residence) and sociodemographic (e.g. age, gender, commitment to environmental protection) conditions [4-5], which cause the existing odour in a given area to become annoying. Therefore, in addition to the sensory methods of odour testing, it is necessary to use methods in which it is important to assess the subjective reception of the odours for people living in the area exposed to an odour. Field studies of odour properties

\footnotetext{
* Corresponding author: izabela.sowka@pwr.edu.pl
} 
(frequency and intensity) together with the help of a qualified team allow objectively assessing the presence of odours characteristic of a given area [6]. Sociological research, in turn, allows carrying out the evaluation of odour nuisance at the same time and in the same area [7]. In the analysis of odour air quality, questionnaire surveys (written, oral and telephone surveys) and individual odour observation diaries are used. Although they have a similar purpose and structure, they differ significantly. Surveys are conducted on a random basis and do not require respondents to involve themselves or their time very much. Odour observation diaries, on the other hand, are filled out voluntarily - the respondents independently collect them and fill them in. In addition, this process requires longer and regular involvement of the respondents [7]. The combination of field and sociological research allows providing results for analysis from two different areas: the objective occurrence of the odour, as well as the subjective assessment by the residents directly exposed to it. As a result, it is possible to indicate the source or sources of the odour, analyse the nuisance (not only related to odours) present in the area and, by analysing the results of the smell observation diaries, estimate the scale of the existing problem.

The aim of the research was to analyse the possibility of using field studies and sociological research to assess the odour air quality and the degree of odour nuisance. The research conducted simultaneously allowed verification of the results used in the evaluation of odour nuisance, which were obtained from the observation of odours by residents of the studied area.

\section{Methodology and research area}

Field measurements were carried out in order to determine the occurrence and intensity characteristic of the examined area. They were conducted on the basis of the German VDI 3940 series guidelines [6,8]. The measurement area consisted of "measurement points" located on the so-called "measuring grid". According to the field research methodology used in European countries, the first stage of measurements was the determination of the research area. In order to do this, a field reconnaissance covering areas of complaints about odours and areas of impact of potentially odorous plants was done first. On this basis, the measuring area and measuring grid consisting of 133 points, spaced by $400 \mathrm{~m}$ (Figure 1), potential odour emission sources (Table 1) and characteristic types of odours (Table 2) were selected and then classified accordingly in odour observation diaries and measurement protocols (Table 2). The questions contained in the diaries concerned the type and source of the odour, the assessment of the degree of odour nuisance in the form of a so-called thermometer scale [8], prevailing meteorological conditions and the duration of the 'odour situation'.

In the measurement protocol, different odour types were given symbols from A to J (Table 2) and an odour intensity scale from 0 to 6 (in accordance with [6]), where the following number indicate respectively: 0 - no odour, 1 - very weak odour, 2 - weak odour, 3 - distinct odour, 4 - strong odour, 5 - very strong odour and 6 - extremely strong odour.

Field studies were conducted by a group of 9 experts and a coordinator. A group of experts has been selected in accordance with PN-EN: 13725 "Air quality. Determination of the olfactory concentration by dynamic olfactometry" [9] using a reference substance (n-butanol in nitrogen). In order to guarantee adequate accuracy in the tests, the duration of a single measurement was 10 minutes and measurements at a given measurement point were performed at least three times, at different times of the day (morning, day, evening or night), by a different person. Moreover, points were randomly assigned to the expert and the date and time of measurement. During the measurement, the person taking the measurement (a member of the expert group), was directed to the wind direction, inhaled 
the surrounding air and noted the smell and type using the appropriate code on the protocol every 10 seconds. Additionally, the basic meteorological data (temperature, humidity, wind speed and direction, sky cover, precipitation) were included into the measurement protocol. In addition, the intensity of the occurring odours was determined during the measurements. Based on the measurements taken, it was possible to determine the frequency of occurrence of a particular type of smell in the so-called odour hours.

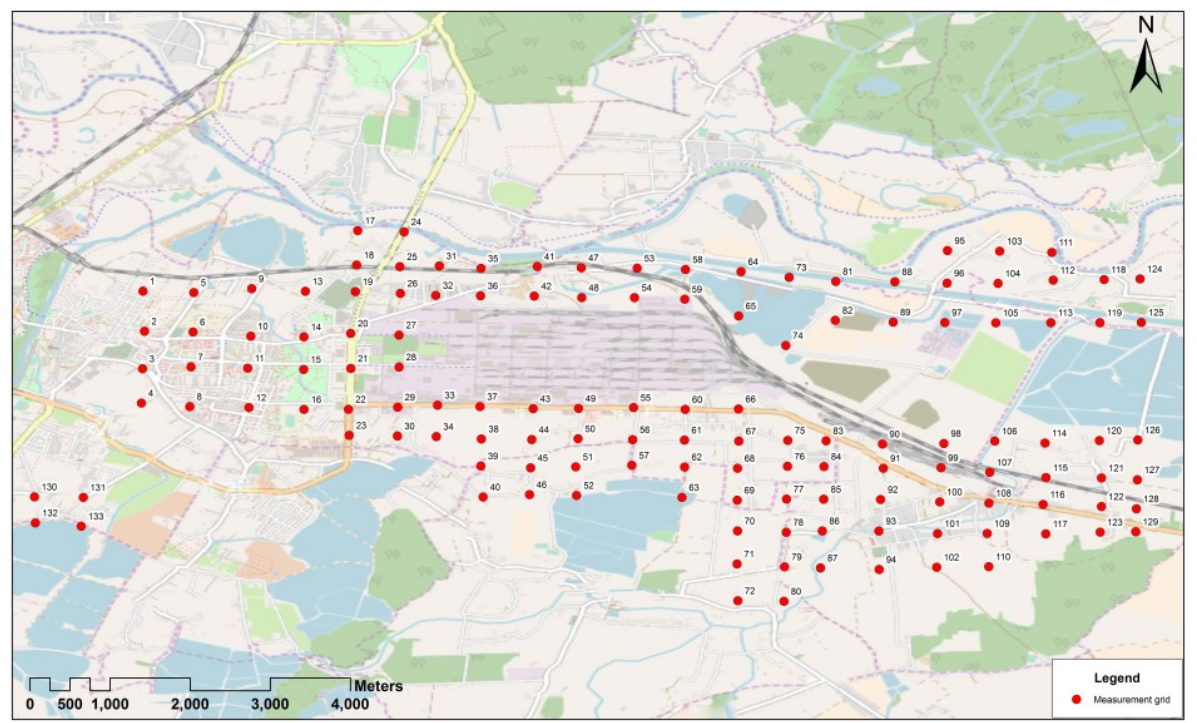

Fig. 1. Location map of the study area (data source for base map layout: [10]).

Table 1. Summary of the potential odour emission sources listed in the odour observation diaries.

\begin{tabular}{|c|c|}
\hline Odour source & Symbol \\
\hline Waste management plant & 1 \\
\hline Means of transport & 2 \\
\hline Industrial plant & 3 \\
\hline Wastewater treatment plant & 4 \\
\hline Material handling & 5 \\
\hline Other sources & 6 \\
\hline
\end{tabular}

Table 2. List of the characteristic types of fragrances listed in the Odour Observation Diaries along with their designations used in the measurement protocols used during field measurements.

\begin{tabular}{|c|c|}
\hline Odour type & Symbol \\
\hline No odour & $\mathrm{A}$ \\
\hline $\begin{array}{c}\text { Industrial (characteristic / } \\
\text { dominating sector of } \\
\text { research) }\end{array}$ & $\mathrm{B}$ \\
\hline Smell of glue & $\mathrm{C}$ \\
\hline Wine & $\mathrm{D}$ \\
\hline Acetic acid & $\mathrm{E}$ \\
\hline Wastewater/industrial & $\mathrm{F}$ \\
\hline Industrial & $\mathrm{G}$ \\
\hline Municipal waste & $\mathrm{H}$ \\
\hline Composting & $\mathrm{I}$ \\
\hline Other & $\mathrm{J}$ \\
\hline
\end{tabular}

In order to assess the degree of odour nuisance in the study area, an "Odour Observation Diary" was also developed. In the prescribed 3-month period coinciding with the field 
research, respondents voluntarily completed one page of the Diary (questionnaire). The potential source (Table 1) and perceived smell type (Table 2) were defined based on the questions contained in the questionnaire form. There were 92 questionnaires for each diary, assigned to specific days.

In order to compare the results of field studies in the range of types and intensity of odours for the maximum values averaged for days on which measurements were conducted, spatial analyses were carried out using geospatial methods in the GIS (geographic information system, GIS: geographic information system, GIS ArcMap 10.3), especially including: designing a measurement grid for the studied area $(5 \times 9 \mathrm{~km})$, as well as determining the maximum and average values of the intensity of the odours studied.

\section{Research and discussion}

\subsection{Odour observations results}

Smell observations were conducted by the inhabitants of the studied area in the Odour Observation Diaries. 36 out of 80 diaries distributed among the residents were completed, and 17 contained complete surveys. Figure 2 shows the location of the observation network and odour observation diaries.

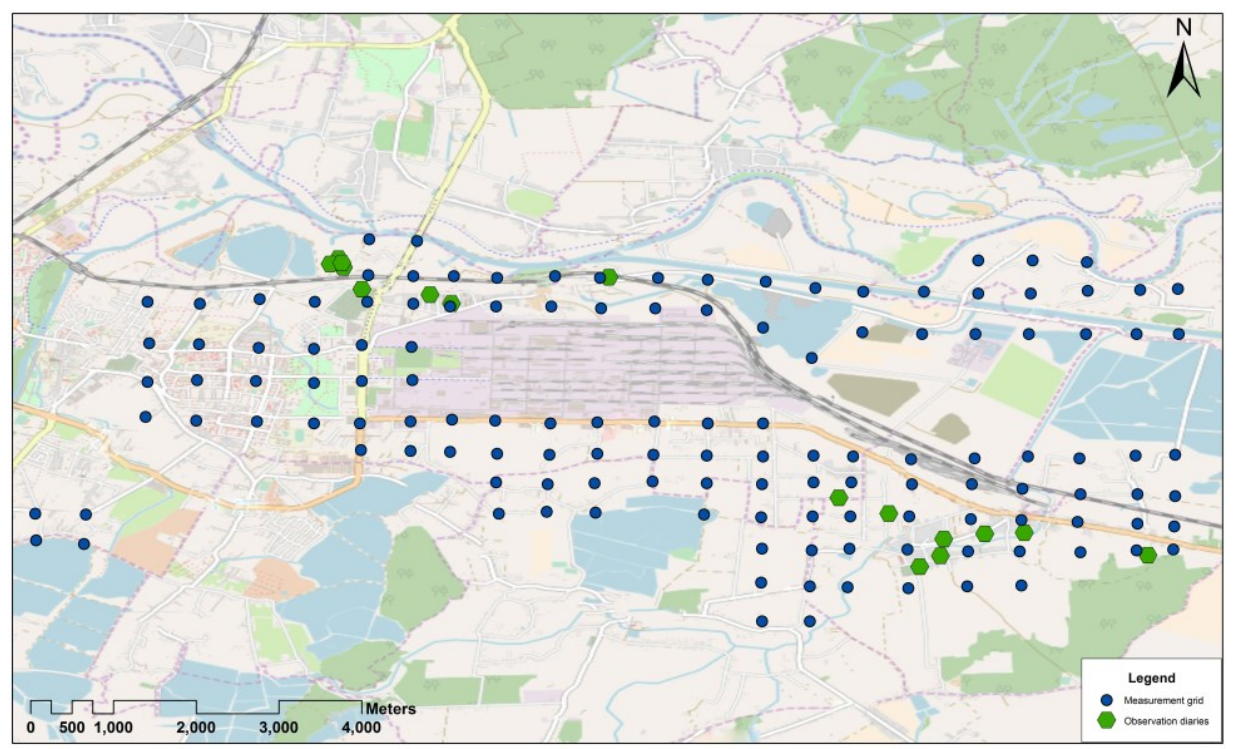

Fig. 2: Location map of the studied area including measuring points in which field measurements were carried out and locations in which Odour Observation Diaries were completely filled in (data source for base map layout: [10]).

The first question in the Diary's questionnaire concerned the duration of a smell in case it occurred. The most frequent response (71 times) was a duration up to $30 \mathrm{~min}$. The second question concerned an indication of the probable source of the odour. Mostly, the likely odour source specified in Table 1 was source no. 4 (36.5\% of responses). In $27 \%$ of the cases the source was indicated as no. $2 ; 12.88 \%$ of the answers indicated the so-called 'other' source from outside; and slightly more than $12 \%$ of the residents indicated odour nuisance generated by source 3 . In $10.3 \%$, the most probable source of the smell was a plant in the municipal sector - source 1. Most frequently, respondents observed the odours marked in Table 2 as: 'G' (38.10\%) and 'F' (27.62\%). The following types of odours were 
indicated as follows: 'H' (8.57\%), 'I' (8.10\%) and the odour referred to as 'J' (8.10\%). $7.62 \%$ of respondents indicated the smell as 'B'. The least responses $(0.95 \%)$ related to a smell defined as 'C' and 'D'. Smell 'E' has not been recorded.

\subsection{Field measurements results}

The research and analyses carried out show that the smells referred to as 'B' and ' $\mathrm{C}$ ' affected the largest area. Nevertheless, the intensity reached its highest values near the tested plant ( 3 - distinct, for the smell $\mathrm{C}$ and even 5 - very strong, in the case of the smell B). The impact of smell $\mathrm{F}$ included a smaller area. It occurred mainly to the south and east of the object from the sector of water and sewage management located in this region. Smell $\mathrm{H}$ occurred mainly (with the highest average intensity of almost 5 - very strong) to the north and north-east of the waste management plant. The average intensity of the smells I, D, E and $G$ reached the intensity value of 3 on the intensity scale used (distinct odour); however, it appeared occasionally in the study area.

Table 3 presents the results of the average intensity of the 8 types of odour present and the average and highest frequency of odour occurrences in the area of all measurement squares.

Table 3. Results of measurements on odour intensity and frequency.

\begin{tabular}{|c|c|c|c|}
\hline Odour type & Odour intensity & Mean frequency, $\%$ & Highest frequency, $\%$ \\
\hline B & 1,7 & 6 & 29 \\
\hline C & 2,2 & 4 & 33 \\
\hline D i E & 1,7 & 0,3 & 4 \\
\hline F & 2,0 & 3 & 19 \\
\hline G & 3,0 & 0,1 & 4 \\
\hline H & 2,3 & 3 & 27 \\
\hline I & 1,9 & 2 & 17 \\
\hline J & 1,9 & 36 & 67 \\
\hline
\end{tabular}

The analysis of the calculations made shows that the most intense smell averaged for the entire measurement period was the ' $G$ ' smell (3 - distinct). The frequency of its occurrence, however, is highest at $4 \%$ during the entire measurement period with the area of occurrence covering one point (two measuring squares), which may indicate a slight impact of this smell on nearby areas. The intensity of other odours varied between 1.7 and 2.3. Also, their average frequency of occurrence did not exceed $6 \%$. The exception is the sum of the remaining smell 'I', where the average frequency of their occurrence was at a level of $36 \%$. The sensation of odour nuisance caused by the occurrence of odours from the above seven groups could therefore be heightened by the presence of such a large share of so-called 'other' odours.

\subsection{Sample results of spatial analysis of test results}

Figures 3 - 5 present examples of spatial analysis results obtained for the odour types B, C and $\mathrm{F}$, and their intensities determined during field measurements from the entire measurement period together with the results of analyses in the odour nuisance assessment carried out by residents using a so-called odour nuisance thermometer scale (17 full-filled Diaries) for the specific places of residence of respondents and points on the measurement grid. 


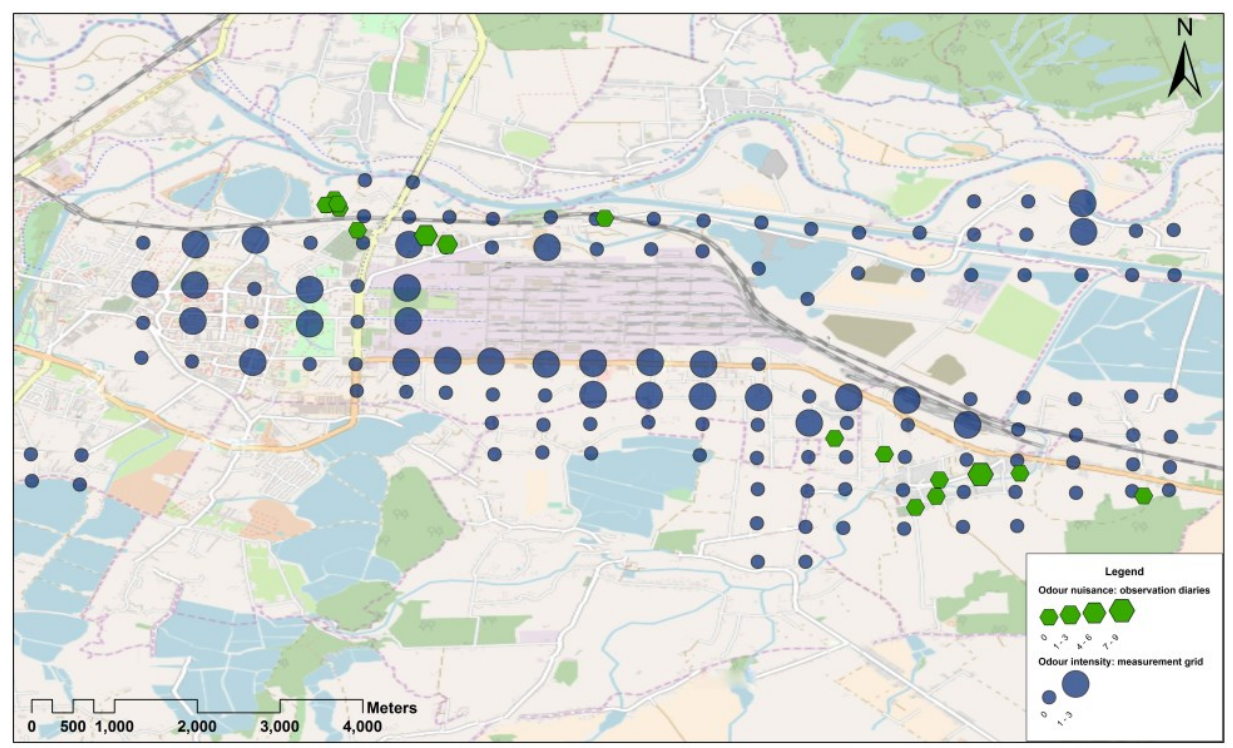

Fig. 3: Example of spatial analysis obtained for odour type 'B' (data source for base map layout: [10]).

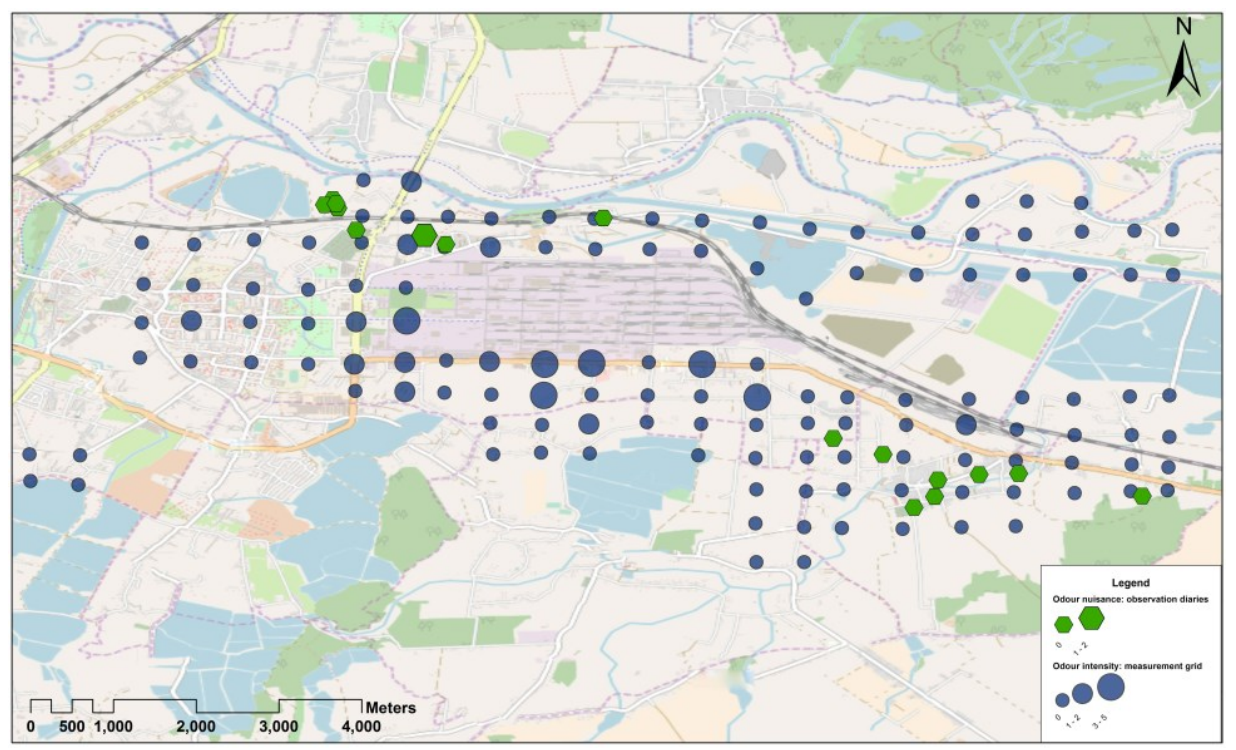

Fig. 4: Example of spatial analysis obtained for odour type 'C' (data source for base map layout: [10]). 


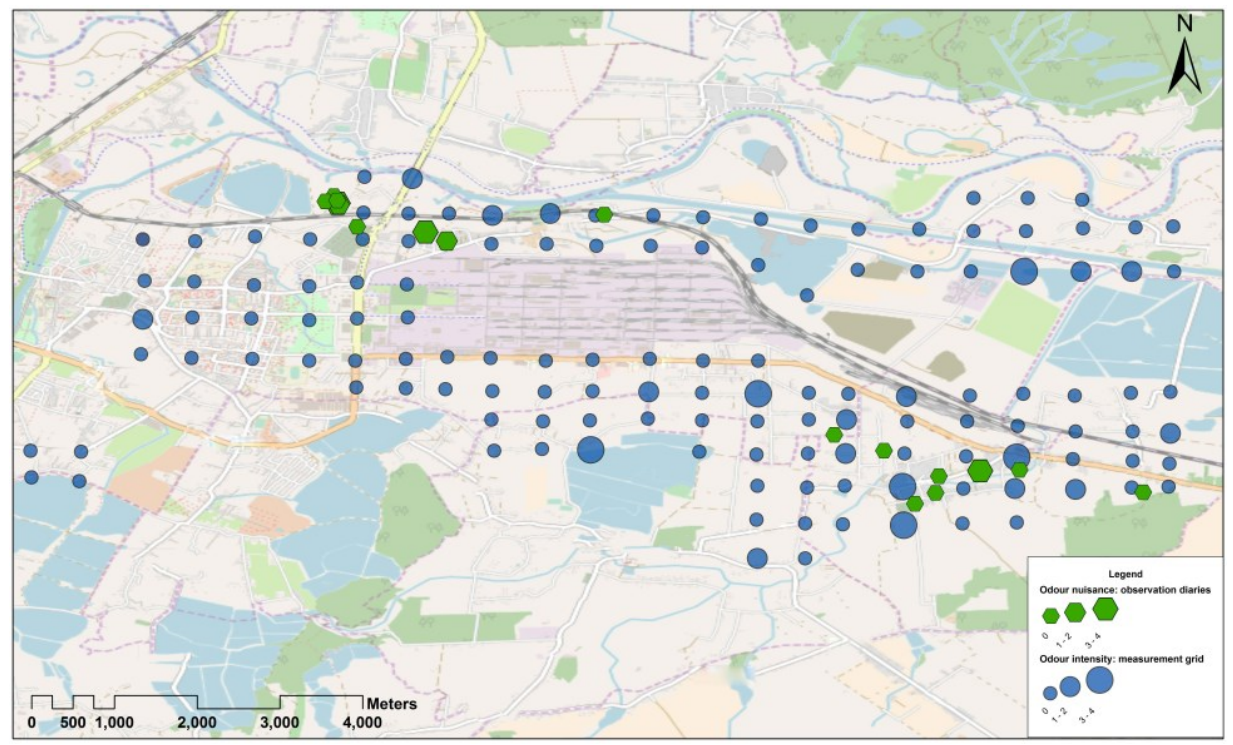

Fig. 5: Example of spatial analysis obtained for odour type 'F' (data source for base map layout: [10]).

The analysis shows that the area affected by the odour covered the immediate vicinity of the industrial plant - situated in the central part of the research area (the specified intensity values reached the highest values: 3 - distinct, smell 'B' and 5 - very strong, smell ' $\mathrm{C}$ ') and plant from the municipal economy sector - in the south-eastern part of the research area (the average odour intensity ranged from 3 - distinct to 5 - very strong odour).

The comparative analysis demonstrates that the average odour intensity values obtained in the way of measurements for selected odours and the specified ranges of the so-called odour nuisance thermometer scale tend to converge in the assessment of air quality and odour - especially in areas located in close vicinity to specific sources of odour emission.

\section{Summary}

Due to the complexity of the topics related to the assessment of the air quality and odour, and the multiplicity of factors affecting the odour and subjective assessment indicating its nuisance in the world and in the European Union, as well as the analytical and sensory methods, methods of sociological research are frequently found, especially in cases of increasing complaints and conflicts. Performed on a long-term scale, e.g. as odour diaries, they can enable observation of the 'odour' situation, indicating the potential source of the odours, their type and duration of 'odour episodes', which in combination with technological information (e.g. nature and type of processes, their duration) and field research - especially in the area subject to the odour influence of several objects - makes possible the identification and determination of the share of individual objects in shaping the aroma situation in the analysed area. In addition, the ability to perform spatial analyses using GIS tools for data obtained, for example, from two types of tests, alongside assessing the occurrence of medium and peaks in intensity of specific types of odours, enables spatial verification of study results obtained from the odour observation diaries, aiming to objectify the assessment of the degree of odour nuisance. 


\section{References}

1. K. Sucker, R. Both, M. Bischoff., R. Guski, U. Kramer, G. Winneke, Int Arch Occup Environ Health. 81(6) (2008)

2. R. Both, K. Sucker, G. Winneke, E. Koch, Water Sci Technol. 50(4) (2004)

3. B. Steinheider, G. Winneke, Journal of Environmental Psychology, 13 (1993)

4. R. Cervinka, E. Neudorfer, Kommission Reinhaltung der Luft im VDI und DIN (Hrsg.): Gerüche in der Umwelt (2007)

5. K. Sucker, R. Both, G. Winneke, Water Science \& Technology 44 (2001)

6. VDI 3940 Parts 1-3, Verein Deutscher Ingenieure, Berlin (2006-2008)

7. VDI 3883, Parts I-II, Verein Deutscher Ingenieure, Berlin (1997)

8. I. Sówka, Scientific Papers of the Institute of Environmental Engineering Wroclaw University of Technology 90 (55) (2011)

9. PN-EN 13725: 2007 Air quality. Determination of odour concentration by dynamic olfactometry (2007)

10. OpenStreetMap.org. Available from. https://github.com/openstreetmap/openstreetmapwebsite/tree/master/config/locales 